\title{
Should we abandon the APTT for monitoring unfractionated heparin?
}

DRJ Arachchillage ${ }^{1,2}$, F Kamani $^{1}$, S Deplano ${ }^{1,}$ W Banya $^{1}$, M Laffan $^{2}$

${ }^{1}$ Department of Haematology, Royal Brompton \& Harefield NHS Foundation Trust, London, UK

${ }^{2}$ Department of Haematology, Imperial College Healthcare NHS Trust and Imperial College London, London, UK

Address for correspondence:

Dr Deepa RJ Arachchillage, Department of haematology, Imperial College Healthcare NHS Trust and Imperial College London, Hammersmith Hospital, 4th Floor, Commonwealth Building, Du Cane Road, London W12 ONN

Tel: +44 (0) 207351 8403, FAX: +44 (0) 2073518402

E-mail: d.arachchillage@imperial.ac.uk

Running title: Monitoring of unfractionated heparin

Abstract word count: 246

Text word count: 2781

Number of Tables and Figures: 2 table, 2 figures 


\begin{abstract}
Introduction

The activated partial thromboplastin time (APTT) is commonly used to monitor unfractionated heparin (UFH) but may not accurately measure the amount of heparin present. The anti-Xa assay is less susceptible to confounding factors and may be a better assay for this purpose.
\end{abstract}

\title{
Materials and Methods
}

The validity of the APTT for monitoring UFH was assessed by comparing with an anti-Xa assay on 3543 samples from 475 patients (infants [ $n=165]$, children 1-15 years [ $n=60]$ and adults [ $n=$ 250]) receiving treatment dose UFH.

\section{Results}

Overall concordance was poor. The highest concordance $(66 \% ; 168 / 254)$ was seen in children. Concordance (51.8\%) or discordance (48.4\%) was almost equal in adult patients. Amongst adult patients whose anti-Xa level was within $0.3-0.7 \mathrm{iu} / \mathrm{ml}$, only $38 \%$ had an APTT in the therapeutic range whilst $56 \%$ were below and $6 \%$ were above therapeutic range. Children and adult patients with anti-Xa of 0.3-0.7 IU/ml but sub- therapeutic APTT had significantly higher fibrinogen levels compared to those with therapeutic or supra-therapeutic APTT.

\section{Conclusions}

When the anti-Xa level was $0.3-0.7 \mathrm{IU} / \mathrm{mL}$, the majority of samples from infants demonstrated a supra-therapeutic APTT, whilst adults tended to have a sub-therapeutic APTT. This may lead to 
under anticoagulation in infants or over anticoagulation in adults with risk of bleeding if APTT is used to monitor UFH. These results further strengthen existing evidence of the limitation of APTT in monitoring UFH. Discordance of APTT and anti-Xa level in adults and children may be due to elevation of fibrinogen level.

\section{Key words}

Heparin, activated partial thromboplastin time, anti-Xa, concordance, fibrinogen 


\section{Introduction}

Unfractionated heparin (UFH) is a sulfated polysaccharide with a molecular weight range of 3000 to $30000 \mathrm{Da}$ (mean, $15000 \mathrm{Da}$ ) and its major anticoagulant action is by inactivating thrombin and activated factor $\mathrm{X}$ (factor $\mathrm{Xa}$ ) through an antithrombin (AT)-dependent mechanism (1). Heparin binds to AT through a high-affinity pentasaccharide, which is present on about a $30 \%$ of heparin molecules. Through its inactivation of thrombin, heparin not only prevents fibrin formation but also inhibits thrombin-induced activation of platelets and of factors V and VIII (1).

UFH has been largely replaced by low molecular weight (LMWH) because the latter is given by subcutaneous injection rather than by intravenous infusion (IV) and has a lower incidence of heparin induced thrombocytopenia and of osteopenia compared to UFH (2). However, for patients undergoing cardiac surgery and in acute intensive care units (AICU) requiring anticoagulation, UFH is still the anticoagulant of choice because of long experience of use in cardio-pulmonary bypass, shorter half-life, independence from renal function, and reversibility by protamine sulphate (2). Compared to LMWH, UFH exhibits a more marked variability in anticoagulant response among individual patients because it is a highly negatively charged molecule and binds to positively charged plasma proteins, proteins released from platelets, and endothelial cell proteins and surfaces $(3 ; 4)$. It is therefore standard practice to adjust the dose of heparin and monitor its effect by measurement of anti-factor Xa activity, activated partial thromboplastin time (APTT) or, when very high doses are used, by the activated clotting time (ACT). 
When the APTT is used for monitoring UFH, it should ideally have the following characteristics; the assay result should have a well-defined and preferably linear relationship with clinical outcome in terms of recurrent thrombosis and bleeding, should have good precision and be well standardized among laboratories and assay reagents and should be readily available and inexpensive $(5 ; 6)$. The widely accepted therapeutic range of activated partial thromboplastin time ratio (APTTR) of 1.5 - 2.5 for UFH therapy is based on a post-hoc analysis of a descriptive clinical study published in 1972 by Basu et al. at McMaster University (7). In this study, it was reported that UFH doses that prolonged an APTT to 1.5-2.5 times control were associated with a reduced risk for recurrent thromboembolism (VTE) based on total of 243 patients which only 162 patients were treated for VTE and the remaining 72 for other diseases. Bleeding occurred in 19 patients whose mean heparin dose and APTT were similar to those of patients without bleeding. Further studies using the "McMaster thromboplastin reagent" demonstrated that the APTTR of 1.5-2.5 corresponded to an UFH level of 0.2 to $0.4 \mathrm{IU} / \mathrm{ml}$ by protamine titration and of 0.3 to $0.7 \mathrm{IU} / \mathrm{ml}$ by anti-Xa assay (8). Although the study by Basu et al (7) was on adults patients, an APTTR of 1.5-2.5 has frequently been accepted as the therapeutic range with different APTT reagents across all age groups, although some centres use an APTT range calibrated against an anti-Xa assay or protamine titration. However, this does not take into account individual patient factors that affect this relationship.

Although the APTT is a cheap and easily available test that is commonly used for monitoring of UFH, it may not provide an accurate measure of the amount of UFH present because of various confounding factors including both pre-analytical and analytical variables. There are three main issues related to APTT when it is used to monitor UFH: 
1. The dose response may vary because UFH binds to other molecules and cells, altering response, and rate of clearance (Glimelius et al, 1978; Mahadoo et al, 1977). Many of these proteins are acute phase reactants and so the degree of this biding can vary between patients and in an individual patient over time.

2. The effect of a given amount of heparin on the APTT varies depending on levels of other coagulation proteins such as factor VIII and fibrinogen; also acute phase reactants (Hirsh et al, 2001).

3. The sensitivity of APTT reagents to heparin varies and there is no system for normalizing this although local calibration can be performed. (9-11). APTT reagents from different manufacturers, and even different batches, show considerable and clinically important variation when heparin concentration by protamine assay is compared with APTT ratio (12).

The anti-Xa assay is not affected by the above factors and has been proposed as a better assay for this purpose. Unlike APTT, use of the anti- Xa assay to monitor UFH does not require reestablishment of the therapeutic range with each new lot of reagent as is necessary for APTT because the recommended anti-Xa therapeutic range of 0.3 to $0.7 \mathrm{IU} / \mathrm{mL}$ does not change. Due to the limitations of APTT in monitoring UFH, it is our standard practice to use anti-Xa level to monitor UFH rather than APTT. Based on comparisons using an anti-factor Xa chromogenic assay within our hospital, an APTT of 60-100 seconds corresponds to the recommended anti-Xa therapeutic range of 0.3 to $0.7 \mathrm{IU} / \mathrm{mL}$. The APTT therapeutic range was determined using 50 patients on stable UFH infusion and compared against the anti-Xa assay to determine the clotting times corresponding to anti-Xa levels between 0.3 and $0.7 \mathrm{iu} / \mathrm{ml}$. These patients 
received UFH only as an anticoagulant and had normal prothrombin time and no more than two samples were collected from the same patient Over this range the APPT and anti-Xa showed a good correlation of $\mathrm{r} 20.64-0.93, \mathrm{p}<0.001$.

Nonetheless, many centres still use APTT to monitor UFH, because it is easily available and less expensive and the aim of this study was to explore the validity of APTT for monitoring UFH in patients of all ages, compared to anti-Xa in patients who are acutely ill.

\section{Patients and methods}

The study was untaken as a service evaluation project in a major tertiary intensive care unit in UK and approved by the trust clinical effectiveness unit. Venous blood was collected into 0.109M trisodium citrate in the proportion 9:1 (Vacutainer Plus, Becton Dickinson, Franklyn Lakes USA), centrifuged at $2000 \mathrm{~g}$ for $10 \mathrm{~min}$ at room temperature and processed within 1 hour of collection. Anti-Xa using chromogenic Liquid anti-Xa assay (Werfen, Warrington, Cheshire, UK), and APTT using SynthASil (HemosIL ${ }^{\circledR}$, Werfen, Warrington, Cheshire, UK) were performed on the same sample using an ACL TOP 500 (Werfen, Warrington, Cheshire, UK). The tests were performed immediately after processing as part of patient management. Clauss fibrinogen level was also performed in the same sample using FIB-C XL kit (Werfen, Warrington, Cheshire, UK) on the ACL TOP 500. The intra- and inter-assay coefficients of variation (CV) were as follows: APTT 2.7\% and 3.0\%; anti-Xa $4.0 \%$ and $6.2 \%$; Clauss fibrinogen $8.0 \%$ and $7.3 \%$ respectively. Patients with coagulation factor deficiencies and lupus anticoagulant were excluded from this study (by investigating patients who had prolonged baseline APTT and excluding patients with known coagulation factor deficiency or lupus anticoagulant). From July 
2016 to December 2016 a total of 3543 samples from 475 patients (infants < 1 year [ $n=165$ ], children 1-16 years $[n=60]$ and adults $[n=250])$ receiving UFH were studied. The majority of the infants and children had congenital heart disease and the majority of the adults were receiving extracorporeal membrane oxygenation (ECMO) for severe respiratory failure or cardiac failure or both.

\section{Statistical analysis}

Data analysis was performed using Stata version 14 and GraphPad Prism ${ }^{\circledR}$ version 7 (GraphPad Software, Inc. La Jolla, USA). Results were reported as median or mean based on the distribution of results with $95 \%$ confidence interval $(\mathrm{Cl})$. Concordance of the APTT with anti-Xa level was assessed using the chi-squared test. Concordance was defined as an APTT in a range appropriate for the corresponding anti-Xa level; i.e. anti-Xa $<0.3 \mathrm{U} / \mathrm{mL}$ and APTT < 60sec; antiXa $0.3-0.7 \mathrm{U} / \mathrm{mL}$ and APTT 60-100 sec and anti-Xa $>0.7 \mathrm{U} / \mathrm{mL}$ and APTT $>100 \mathrm{sec}$. As there were repeated measurements of the same patients, linear regression was not performed. However, the relationship between APTT and anti-Xa in the three age groups is presented graphically. (Figure $1 \mathrm{~A}-\mathrm{C}$ )

Patients who had anti-Xa levels within the therapeutic range $0.3-0.7 \mathrm{u} / \mathrm{ml}$ were selected and their fibrinogen levels were compared with the APTT levels using a linear mixed model for the comparison. The lowest level of APTT $(<60 \mathrm{sec})$ was used as the baseline comparator for those patients with APTT $60-100 \mathrm{sec}$ and $>100 \mathrm{sec}$. A p value of $<0.05$ was considered significant. 


\section{Results}

Figure $1(A-C)$ illustrates the relationship between APTT and anti-Xa in the three age groups.

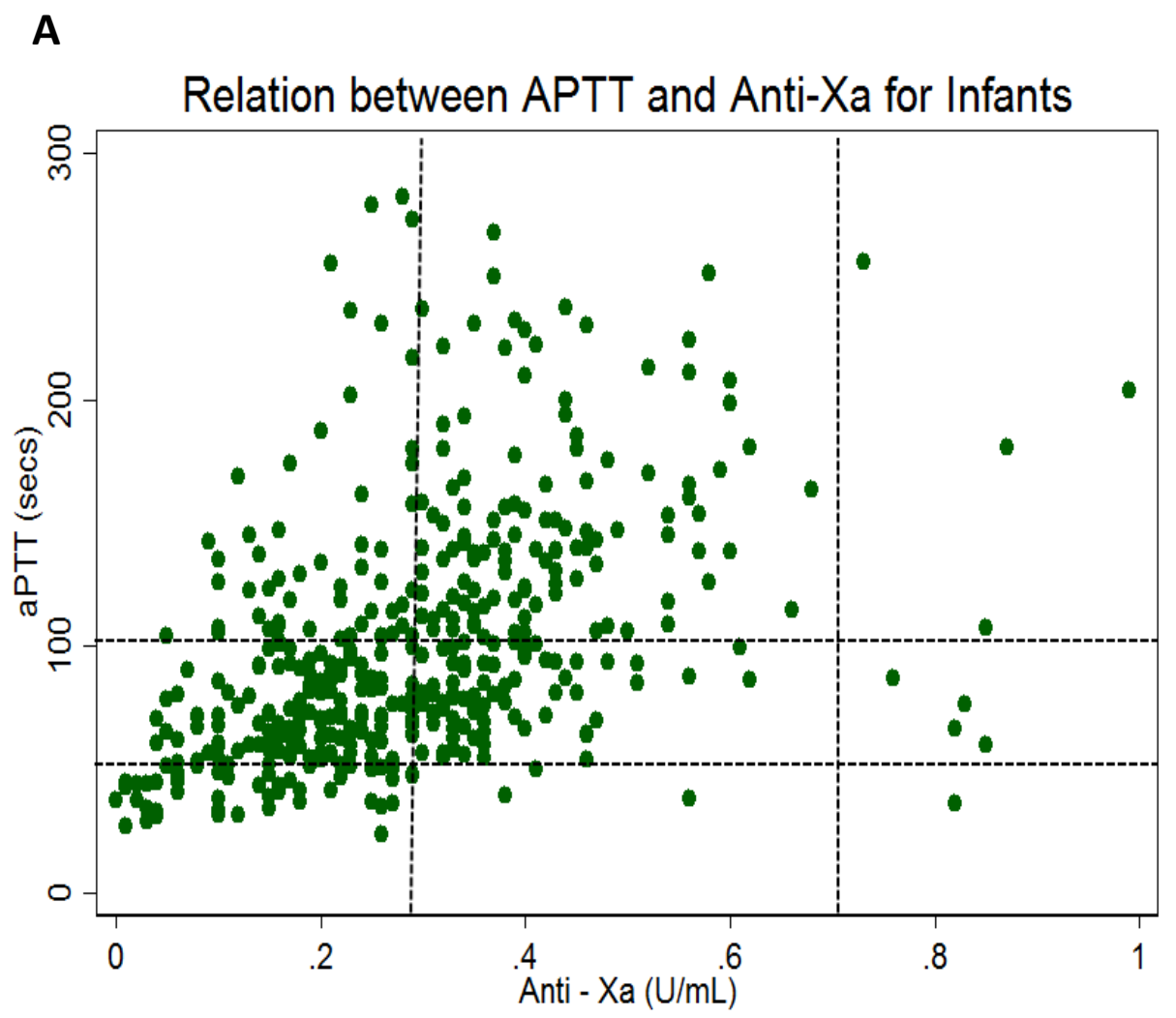


B

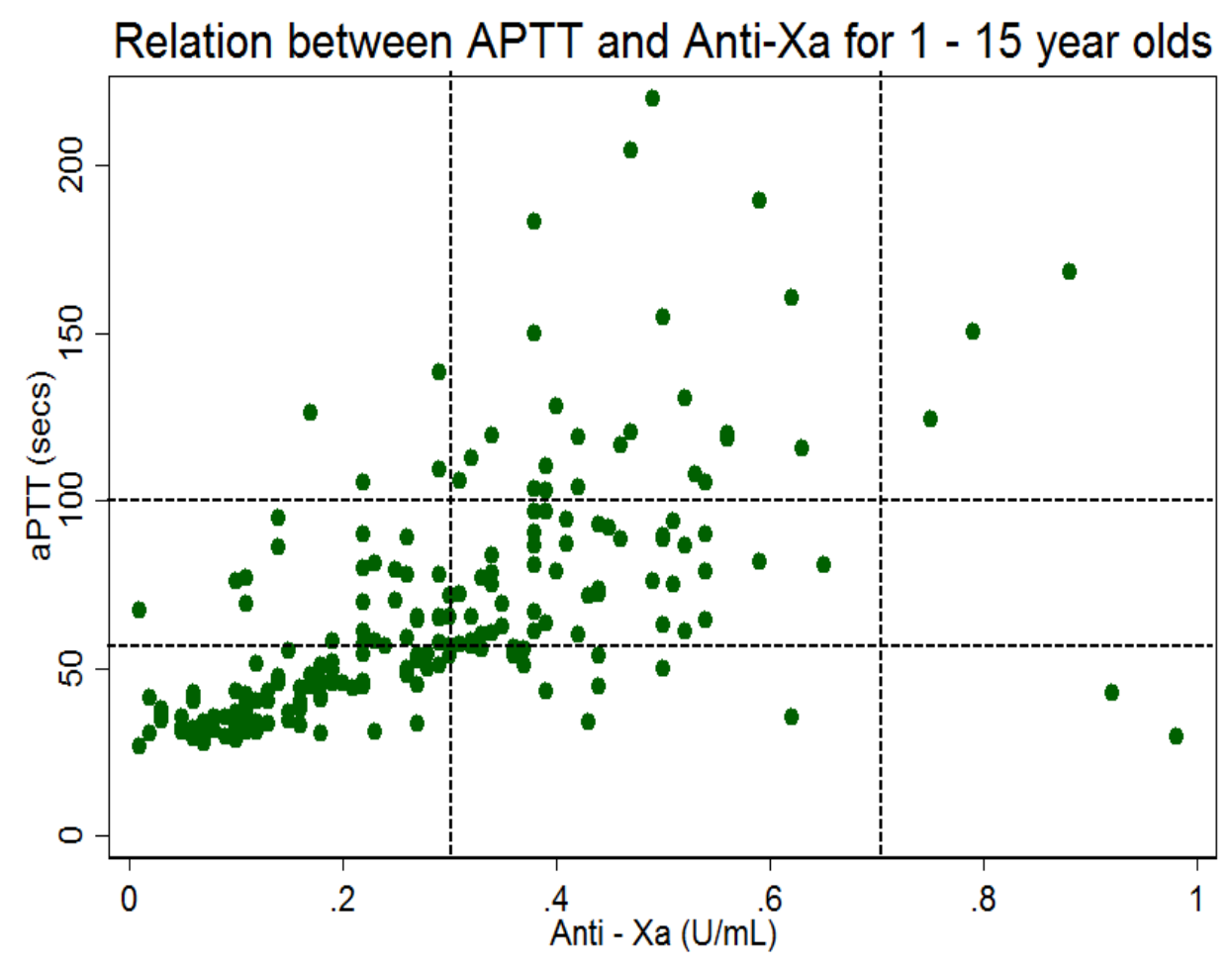


C

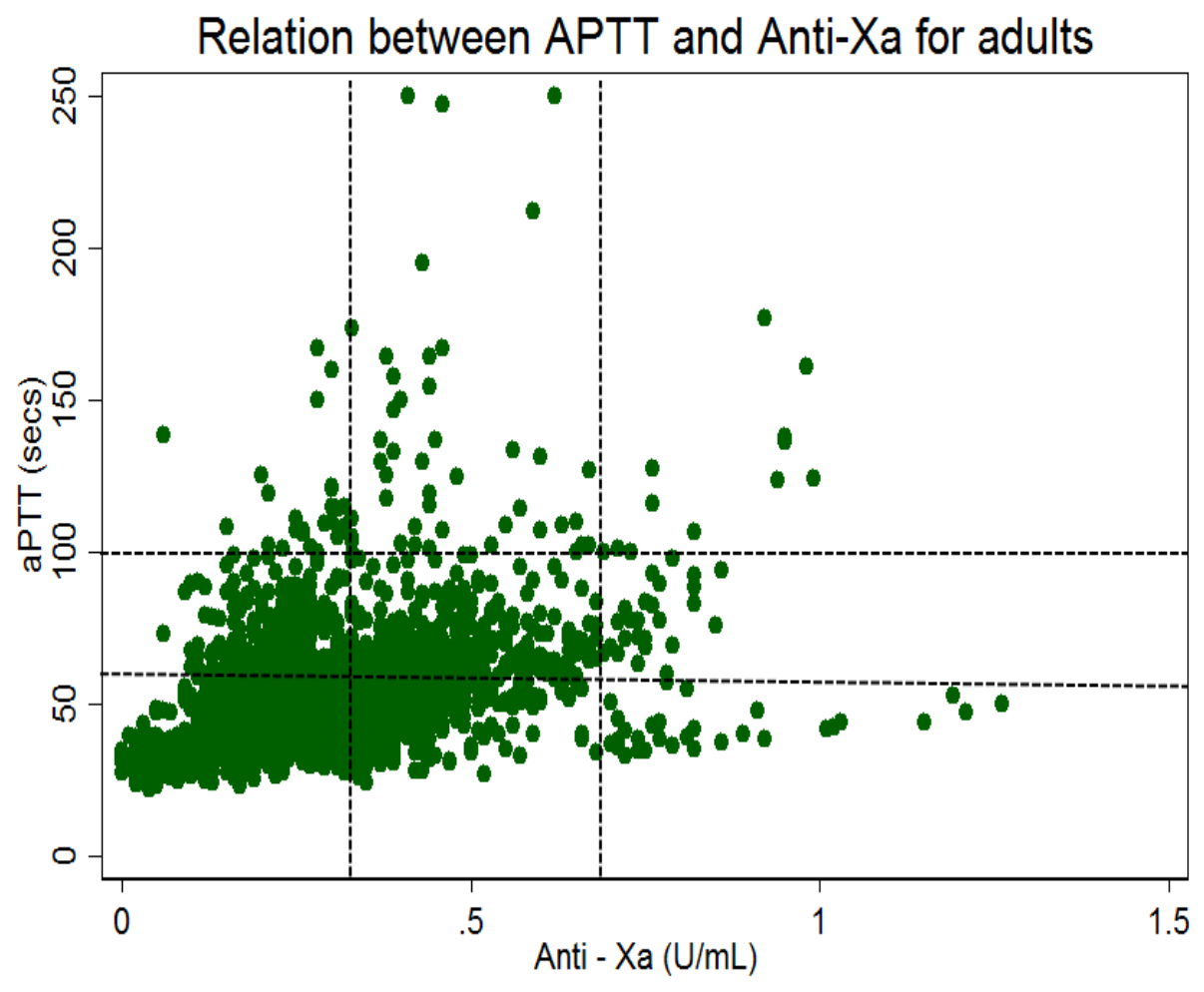

Dotted lines in each graph indicate the therapeutic range of APTT $(60-100 \mathrm{sec})$ and anti-Xa $(0.3-$ $0.71 \mathrm{U} / \mathrm{mL}$ ) levels 
Table 1 summaries the relationship according to the anti-Xa level for each of the age groups.

For samples with anti-Xa levels of $0.3-0.7 \mathrm{u} / \mathrm{mL}$, there was a wide range of APTT results (mean 62 , range 29-250seconds) observed.

Table 1

\begin{tabular}{llllll}
\hline & $\begin{array}{l}\text { Anti-Xa } \\
\text { IU/mL }\end{array}$ & $\begin{array}{l}\text { No.(\%) of } \\
\text { samples in the } \\
\text { sub-therapeutic } \\
\text { range (APTT } \\
<60 \text { s) }\end{array}$ & $\begin{array}{l}\text { No.(\%) of } \\
\text { samples in the } \\
\text { therapeutic } \\
\text { range (APTT 60- } \\
100 s)\end{array}$ & $\begin{array}{l}\text { No.(\%) of } \\
\text { samples in the } \\
\text { supra- } \\
\text { therapeutic } \\
\text { range (APTT } \\
>100 s)\end{array}$ & Total \\
\hline Infants & $<0.3$ & $85(34 \%)$ & $100(40 \%)$ & $64(26 \%)$ & 249 \\
(<1year) & $0.3-0.7$ & $10(5 \%)$ & $60(31 \%)$ & $123(64 \%)$ & 193 \\
\hline Children & $>0.7$ & $2(12 \%)$ & $4(23 \%)$ & $11(65 \%)$ & 17 \\
(1-15 years) & $0.3-0.7$ & $20(17 \%)$ & $55(48 \%)$ & $40(35 \%)$ & 115 \\
& $>0.7$ & $2(20 \%)$ & $3(30 \%)$ & $5(50 \%)$ & 10 \\
\hline Adults & $<0.3$ & $1370(84 \%)$ & $251(15 \%)$ & $17(1 \%)$ & 1638 \\
$(16-88$ years) & $0.3-0.7$ & $640(56 \%)$ & $432(38 \%)$ & $64(6 \%)$ & 1136 \\
& $>0.7$ & $12(21 \%)$ & $23(41 \%)$ & $21(38 \%)$ & 56 \\
\hline
\end{tabular}

The overall concordance of the APTT with anti-Xa levels in the three patient groups is shown in Table 2. The highest concordance (66\%; 168/254) was seen in children age 1-15 years. The highest discordance (67.6\%; 310/459) was seen infants. Of the 193 samples from 165 infants with heparin anti-Xa levels of $0.3-0.7 \mathrm{IU} / \mathrm{mL}, 10(5 \%), 60$ (31\%) and $123(64 \%)$ had an APTT in the sub-therapeutic, therapeutic (60-100s) and supra-therapeutic range respectively. Concordance 
(1466/2836 [51.8\%]) or discordance (1370/2836 [48.4\%]) of APTT and anti-Xa in adult patients were almost equal. However, amongst adult patients, whose anti-Xa level was within 0.3-0.7 $\mathrm{iu} / \mathrm{ml}$, only $38 \%$ had an APTT in the therapeutic range whilst $(56 \%)$ were below and $6 \%$ were above the therapeutic range.

Table 2.Concordance of APTT with anti-Xa levels

\begin{tabular}{|l|l|l|l|}
\hline & $\begin{array}{l}\text { Infants } \\
(<1 \text { year })\end{array}$ & $\begin{array}{l}\text { Children } \\
(1-15 \text { years })\end{array}$ & Adults (16-88years) \\
\hline Discordance & $310(67.6 \%)$ & $86(34 \%)$ & $1370(48.4 \%)$ \\
\hline Concordance & $149(32.4 \%)$ & $168(66 \%)$ & $1466(51.8 \%)$ \\
\hline
\end{tabular}

Children and adult patients with anti-Xa of $0.3-0.7 \mathrm{IU} / \mathrm{ml}$ but sub-therapeutic APTT had significantly higher fibrinogen levels compared to those with therapeutic range APTT (60100sec) and supra-therapeutic APTT (>100sec) [Figure 2 B and C]. Mean fibrinogen and confidence interval (Cl) for children were 4.06g/L (3.64-4.49) for APTT <60sec vs 3.5g/L (3.153.84) for APTT 60sec, $p=0.002$ and 3.38g/L (2.92-3.84) for APTT > 100sec, $p=0.004)$. For adult patients the mean fibrinogen and $\mathrm{Cl}$ were 4.69/L (4.55-4.82) for APTT <60sec vs 4.40g/L (4.304.50) for APTT 60sec, $p<0.0001$ and 3.73g/L (3.51-3.98) for APTT $>100$ sec, $p<0.0001$ (Figure 2 B and C). There was no significant difference in the fibrinogen levels in infants with anti-Xa of 0.3$0.7 \mathrm{IU} / \mathrm{ml}$ but sub-therapeutic APTT [2.83 (2.43-3.20)] compared to those with therapeutic [2.88 (2.49-3.19), $p=0.89$ ] and supra-therapeutic APTT [2.63 (2.27-3.0), $p=0.29$ ] (Figure 2 A). 
Figure 2. Fibrinogen levels in patients with sub-therapeutic APTT compared to those with therapeutic range APTT $(60-100 \mathrm{sec})$ and supra-therapeutic APTT $(>100 \mathrm{sec})$

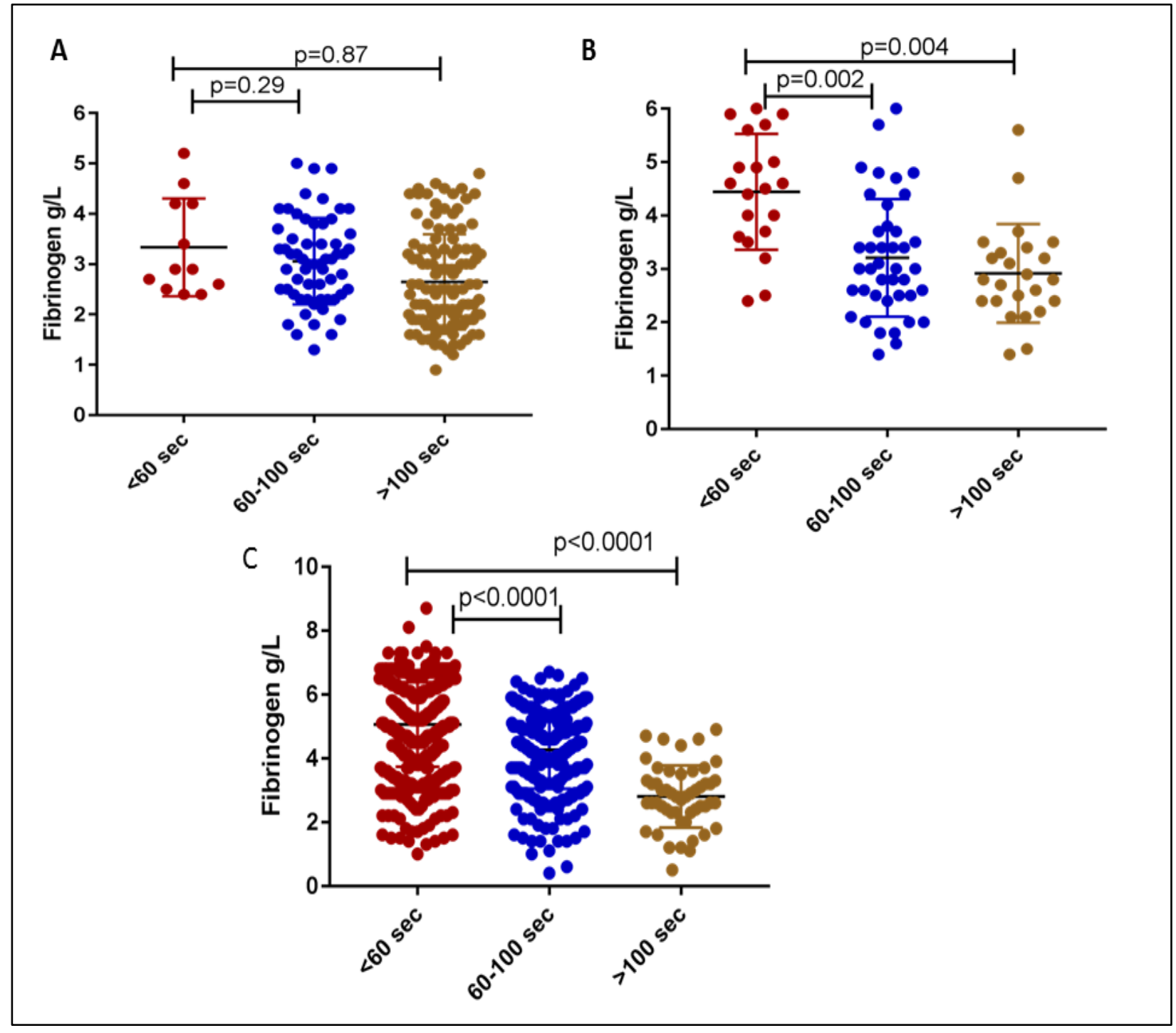

\section{Discussion}

We report the largest study to date investigating the concordance of anti-Xa and APTT in monitoring therapeutic dose UFH across all age groups. This study demonstrated that there was 
a poor concordance between APTT and heparin anti-Xa levels in all age groups of patients treated with UFH in intensive care settings. Concordance was poor in patients with extremes of ages (infants and adults) whilst the highest concordance (66\%; 168/254) was seen in children age 1-15 years. Infants tend to have more supra-therapeutic values of APTT with subtherapeutic values of heparin anti-Xa whilst adult patients had more sub-therapeutic APTT with therapeutic heparin anti-Xa values. This is in keeping with delayed development of the coagulation systems in infants in which production of vitamin $\mathrm{K}$ dependent factors is not complete until 6 months of age (13). In adults plasma concentrations of some coagulation proteins such as factor V, VIII, IX and fibrinogen increase progressively with age, which may contribute to shorter APTT (14). In our study children and adult patients with anti-Xa of 0.3-0.7 IU/ml but sub-therapeutic APTT had significantly higher fibrinogen levels compared to those with therapeutic range APTT (60-100sec) and supra-therapeutic APTT (>100sec). This was not seen in infants suggesting that there must be other explanations for the discordance, which is understandable as another aspect of the developing coagulation system. Association of heparin resistance with elevated levels of fibrinogen has been reported long time ago even before the studies investigating the APTTR to monitor UFH (Edson et al, 1967). In a previous study of 569 samples from 149 patients on UFH it was found that anti-Xa values and the APTT were concordant in only $54 \%$ of measurements (15). A sub group of 59 patients samples were tested for factor II, factor VIII, and endogenous thrombin potential (ETP) in addition to APTT and antiXa assays. In patients with supra-therapeutic APTT but therapeutic anti-Xa, there was decreased factor II activity whilst those patients with sub-therapeutic APTT but therapeutic anti-Xa values had high factor VIII activity. The total amount of thrombin generated (ETP) 
(assessed in a calibrated automated thrombogram) was inversely correlated with anti-Xa and UFH dose (15). Several studies have reported the discordance of the APTT with anti-Xa values in monitoring heparin (15-19) and many studies have focused on one particular age group, either infants $(18)$ or adults $(16 ; 17 ; 19)$. One study demonstrated that nearly $57 \%$ of patients in the anti-Xa group were in range within 6 hours of initiation of UFH versus $27 \%$ in the APTT group (p $=0.001$ (20). Patients in the anti-Xa group had an average of 1.00 dosage adjustments per subject compared to 1.71 in the APTT group within the first 24 hours $(p=0.003)(20)$. A study by Price et al, 2013 using 2321-paired APTT and anti-Xa values from 539 adults demonstrated 937 (40\%) concordance of APTT and anti-Xa values (19). Importantly, they found that patients with two or more consecutive high APTT compared to anti-Xa values had significantly increased 21day major bleeding ( $9 \%$ vs $3 \% ; p=0.0316)$ and 30 -day mortality $(14 \%$ vs $5 \%) ; p=0.0202$ ) rates compared with patients with consistently concordant values. This implies that the anti-Xa is a more clinically relevant measure of anticoagulation, even though the APTT is a global assay. In another study by Adatya et al, using 340-paired values from 38 patients, it was shown that concordance for anti-FXa and APTT was as low as 24.6\% (87/340) (17). Findings from previously reported studies investigating the concordance of APTT and anti-Xa levels in infants are in keeping with our results (18).

In contrast to our data on adult patients, where a discordantly low APTT (640/1136 [56\%]) was the most common abnormality, a disproportionate prolongation of the APTT relative to the anti-Xa was the most common discordant pattern in the above studies(17;19). This could be due to the difference in study population: the majority of our patients were on ECMO and will have had an acute inflammatory response; compared to various other indications including 
patients receiving continuous-left flow ventricular assist devices and venous thromboembolism in other studies. In addition, other studies did not systematically exclude patients with prolonged APTT due to coagulation factor deficiency or lupus anticoagulant. In our study, we have studied all age groups and considered the influence of fibrinogen on the APTT reflecting the acute inflammatory response.

Unlike APTT, the anti- Xa assay does not require re-establishment of the therapeutic range with each new lot of reagent because the recommended therapeutic range of 0.3 to $0.7 \mathrm{IU} / \mathrm{mL}$ does not change. Although the per-test cost of anti-Xa is twice that of the APTT, many studies show that anti-Xa measurement achieved therapeutic anticoagulation more rapidly, maintained the values within the target range for longer and required fewer repeat tests and dose adjustments compared to APTT monitoring $(20 ; 21)$. In our study, the median cost per-test for APTT was $f 6$ compared to $f 13$ for anti-Xa (including the costs for reagents, biomedical scientist time, equipment and maintenance. However, with the use of anti-Xa to monitor UFH, the average number of heparin dose changes was lower. On average, patients monitored with Anti-Xa had fewer tests (4.5) than patients those monitored with aPTT (7.21), $\mathrm{p}<0.001$. It is therefore likely that the anti- $\mathrm{Xa}$ is a cost-effective method for monitoring UFH. Furthermore, the anti-Xa assay is quicker to perform ( 2 mins) compared to APTT ( 6mins) once the analyser is calibrated. Thus, in contrast to the common misunderstanding about cost and turnaround time, there is little reason for laboratories not to offer anti-Xa assays as a routine test available over $24 \mathrm{hrs}$.

Indeed, it is our standard practice to use anti-Xa rather than APTT to monitor the anticoagulant effect of UFH and this study was not designed to determine the clinical consequences of APTT monitoring. When a significant discordance between the two parameters (especially in 
appropriately high APTT) was detected, further investigations were performed to rule out an underlying bleeding tendency and close monitoring for evidence of bleeding undertaken. However, we would be concerned that if the APTT were used for monitoring of UFH, a significantly large number of patients would be at increased risk of bleeding or thrombosis due to over or under anticoagulation in all age groups. We agree that laboratories should at least establish their own APTT therapeutic range as recommend by both the American College of Chest Physicians (22) British Committee for Standards in Haematology (23).

It is important to note that during cardiac surgery, much higher concentrations of heparin are used which are usually monitored using the activated clotting time (ACT). We have not examined the relationship between high levels of heparin and the ACT in this study and so cannot comment on monitoring in this situation. In addition, although factor VIII level is also often elevated in patients treated in intensive care units due to underlying infections/inflammation, this was not assessed in majority of patients included in this study.

In conclusion, concordance was poor between anti-Xa level and APTT in all age groups and it was worse in infants. The majority of samples from infants demonstrated a supra-therapeutic APTT, whilst adults tended to have a sub-therapeutic APTT, when the anti-Xa levels were 0.3$0.7 \mathrm{U} / \mathrm{mL}$. Dose adjustments based on the APTT will therefore lead to under anticoagulation in infants or over anticoagulation in adults with consequent risk of thrombosis or bleeding. These results further strengthen the existing evidence indicating the limitations of APTT monitoring of UFH therapy. Under-responsiveness of the APTT and anti-Xa level in adult and children may be partly accounted by elevated fibrinogen levels secondary to an acute phase reaction. Over- 
responsiveness in infants may reflect an immature coagulation system. Individual Trusts should evaluate their current practice based on their specific local populations to consider whether the anti-Xa assay is a better option than APTT for monitoring UFH in terms of both clinical safety and cost effectiveness.

\section{Acknowledgements}

We would like to thank S Fox, S Davidson and M Mcevoy and coagulation laboratory staff at Royal Brompton Hospital, London for performing the assays.

\section{Authorship}

DRJA was involved in study concept and design, data collection, analysis and interpretation of data, and prepared the first draft of the manuscript. FK performed part of the assays, supported in data collection and revised the manuscript. SD collected the data and revised the manuscript WB analysed the data and revised the manuscript. ML was involved study concept, interpretation of the data and revising the manuscript. All authors approved the final manuscript.

\section{Disclosure of conflict of interest}

Authors state that they have no relevant conflict of interest 


\section{References}

(1) Bussey H, Francis JL. Heparin overview and issues. Pharmacotherapy 2004;24:103S -107S.

(2) Eikelboom JW, Hirsh J. Monitoring unfractionated heparin with the aPTT: time for a fresh look. Thromb Haemost 2006 ; 96:547 -52.

(3) Young E, Podor TJ, Venner T, Hirsh J. Induction of the acute-phase reaction increases heparinbinding proteins in plasma. Arterioscler Thromb Vasc Biol 1997 ;17:1568 -74.

(4) Young E, Venner T, Ribau J, Shaughnessy S, Hirsh J, Podor TJ. The binding of unfractionated heparin and low molecular weight heparin to thrombin-activated human endothelial cells. Thromb Res $1999 ; 96: 373-81$.

(5) Olson JD, Arkin CF, Brandt JT, Cunningham MT, Giles A, Koepke JA, et al. College of American Pathologists Conference XXXI on laboratory monitoring of anticoagulant therapy: laboratory monitoring of unfractionated heparin therapy. Arch Pathol Lab Med $1998 ; 122: 782$-98.

(6) Kassai B, Shah NR, Leizorovicza A, Cucherat M, Gueyffier F, Boissel JP. The true treatment benefit is unpredictable in clinical trials using surrogate outcome measured with diagnostic tests. J Clin Epidemiol $2005 ; 58: 1042$-51.

(7) Basu D, Gallus A, Hirsh J, Cade J. A prospective study of the value of monitoring heparin treatment with the activated partial thromboplastin time. N Engl J Med 1972 A;287:324 -7.

(8) Hirsh J, Raschke R. Heparin and low-molecular-weight heparin: the Seventh ACCP Conference on Antithrombotic and Thrombolytic Therapy. Chest 2004 ;126 (3 Suppl):188S -203S.

(9) Marlar RA, Gausman JN. The effect of instrumentation and laboratory site on the accuracy of the APTT-based heparin therapeutic range. Int J Lab Hematol $2012 ; 34$ :614 -20.

Favaloro EJ, Bonar R, Sioufi J, Wheeler M, Low J, Aboud M, et al. An international survey of current practice in the laboratory assessment of anticoagulant therapy with heparin. Pathology $2005 ; 37: 234-8$.

(11) Brigden ML, Johnston M. A survey of aPTT reporting in Canadian medical laboratories. The need for increased standardization. Thrombosis Interest Group of Canada. Am J Clin Pathol $2000 ; 114$ :276-82.

(12) Brill-Edwards P, Ginsberg JS, Johnston M, Hirsh J. Establishing a therapeutic range for heparin therapy. Ann Intern Med $1993 ; 119: 104-9$.

(13) Boris J, Harris M. The use of anticoagulation in pediatric cardiac disease. Images Paediatr Cardiol 2003 ; 5:1-35. 
(14) Favaloro EJ, Franchini M, Lippi G. Aging hemostasis: changes to laboratory markers of hemostasis as we age - a narrative review. Semin Thromb Hemost 2014;40:621 -33

(15) Takemoto CM, Streiff MB, Shermock KM, Kraus PS, Chen J, Jani J, et al. Activated partial thromboplastin time and anti-xa measurements in heparin monitoring: biochemical basis for discordance. Am J Clin Pathol 2013;139:450-6.

(16) Mitrovska S, Jovanova S. Correlation between the aPTT and clinical events in acute coronary syndromes treated with unfractionated heparin. Bratisl Lek Listy 2006 ;107 (:422 -5.

(17) Adatya S, Uriel N, Yarmohammadi H, Holley CT, Feng A, Roy SS, et al. Anti-factor Xa and activated partial thromboplastin time measurements for heparin monitoring in mechanical circulatory support. JACC Heart Fail $2015 ; 3: 314-22$.

18) Kuhle S, Eulmesekian P, Kavanagh B, Massicotte $P$, Vegh $P$, Lau A, et al. Lack of correlation between heparin dose and standard clinical monitoring tests in treatment with unfractionated heparin in critically ill children. Haematologica $2007 ; 92554-7$.

(19) Price EA, Jin J, Nguyen HM, Krishnan G, Bowen R, Zehnder JL. Discordant aPTT and anti-Xa values and outcomes in hospitalized patients treated with intravenous unfractionated heparin. Ann Pharmacother $2013 ; 47: 151-8$.

(20) Fruge KS, Lee YR. Comparison of unfractionated heparin protocols using antifactor Xa monitoring or activated partial thrombin time monitoring. Am J Health Syst Pharm 2015;72 (17 Suppl 2):S90 -7

(21) Guervil DJ, Rosenberg AF, Winterstein AG, Harris NS, Johns TE, Zumberg MS. Activated partial thromboplastin time versus antifactor Xa heparin assay in monitoring unfractionated heparin by continuous intravenous infusion. Ann Pharmacother $2011 ; 45: 861-8$.

(22) Hirsh J, Raschke R. Heparin and low-molecular-weight heparin: the Seventh ACCP Conference on Antithrombotic and Thrombolytic Therapy. Chest $2004 ; 126$ :188S -203S.

(23) Baglin T, Barrowcliffe TW, Cohen A, Greaves M. Guidelines on the use and monitoring of heparin. Br J Haematol $2006 ; 133: 19-34$. 